\title{
The Health and Sport Engagement (HASE) Intervention and Evaluation Project: protocol for the design, outcome, process and economic evaluation of a complex community sport intervention to increase levels of physical activity
}

Louise Mansfield, Nana Anokye, Julia Fox-Rushby, Tess Kay

To cite: Mansfield L, Anokye N, Fox-Rushby J, et al. The Health and Sport Engagement (HASE) Intervention and Evaluation Project: protocol for the design, outcome, process and economic evaluation of a complex community sport intervention to increase levels of physical activity. BMJ Open 2015;5:e009276. doi:10.1136/bmjopen-2015009276

- Prepublication history for this paper is available online. To view these files please visit the journal online (http://dx.doi.org/10.1136/ bmjopen-2015-009276).

Received 2 July 2015 Revised 16 September 2015 Accepted 6 October 2015

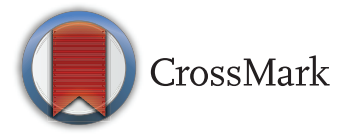

Department of Life Sciences, Brunel University London, London, UK

Correspondence to Dr Louise Mansfield; louise.mansfield@brunel.ac.uk

\section{ABSTRACT}

Introduction: Sport is being promoted to raise population levels of physical activity for health. National sport participation policy focuses on complex community provision tailored to diverse local users. Few quality research studies exist that examine the role of community sport interventions in raising physical activity levels and no research to date has examined the costs and cost-effectiveness of such provision. This study is a protocol for the design, outcome, process and economic evaluation of a complex community sport intervention to increase levels of physical activity, the Health and Sport Engagement (HASE) project part of the national Get Healthy Get Active programme led by Sport England.

Methods and analysis: The HASE study is a collaborative partnership between local community sport deliverers and sport and public health researchers. It involves designing, delivering and evaluating community sport interventions. The aim is to engage previously inactive people in sustained sporting activity for $1 \times 30$ min a week and to examine associated health and well-being outcomes. The study uses mixed methods. Outcomes (physical activity, health, well-being costs to individuals) will be measured by a series of self-report questionnaires and attendance data and evaluated using interrupted time series analysis controlling for a range of sociodemographic factors. Resource use will be identified and measured using diaries, interviews and records and presented alongside effectiveness data as incremental cost-effectiveness ratios and costeffectiveness acceptability curves. A longitudinal process evaluation (focus groups, structured observations, in-depth interview methods) will examine the efficacy of the project for achieving its aim using the principles of thematic analysis.

Ethics and dissemination: The results of this study will be disseminated through peer-reviewed

\section{Strengths and limitations of this study}

- The first mixed-method evaluation of a complex community sport intervention aiming to engage inactive people in sustained sporting activity to promote physical activity, health and well-being.

- A rigorous comparative evaluation and the inclusion of economics, both rare in evaluating sport interventions.

- A strong quasi-experimental design providing high policy interest in the findings.

- Brunel University investigators co-developed the intervention and will evaluate the project requiring continual work to ensure that close observations by those most invested in the project are balanced with the level of independence required for high-quality evidence production.

- The study does not include a control group.

publications, academic conference presentations, Sport England and national public health organisation policy conferences, and practice-based case studies. Ethical approval was obtained through Brunel University London's research ethics committee (reference number RE33-12).

\section{INTRODUCTION}

In the aftermath of the London 2012 Olympic and Paralympic Games, the sport sector is currently one priority area for increasing population rates of physical activity for health. ${ }^{1}$ The Moving More, Living More cross-government group includes representation from Sport England, the Department of Health and Public Health 
England and recognises the role that sport can play in helping people to become more active. There are national ambitions to increase and measure regular participation in sport. ${ }^{2}{ }^{3}$ This study is part of a national Sport England Get Healthy Get Active funding stream which aims to examine the role of sport in engaging previously inactive people in physical activity.

Successive Sport England strategies have focused on developing sporting opportunities tailored to the needs of diverse communities of local users. Together with devolvement of public health priorities to local authorities in April 2013, this heightens the significance of locality and community in intervening to increase physical activity through sport. National sport participation policies with local emphasis can result in quite varied delivery programmes appropriate to different communities. This serves to emphasise expectations that sport participation policy implementation will be through complex interventions at the community level. Complex interventions are most commonly associated with service delivery and practice in health, and in education, transport and housing where health consequences are evident or expected. ${ }^{4}$ These interventions involve several interlocking factors, and a diverse range of participants and organisational groups. ${ }^{56}$

Evidence supporting the link between interventions delivered by sports organisations with increases in sport participation or physical activity is weak. ${ }^{7}$ A recent review of research and practice on improving health through sport participation highlighted a dearth of evidence from high-quality studies. ${ }^{8}$ Moreover, there is a set of mixed results from lower quality studies ${ }^{9-11}$ with even less from community-based studies. ${ }^{12}{ }^{13}$ One longitudinal preintervention and postintervention questionnaire assessment of a national community physical activity pilot project to increase levels of physical activity reported that $58.5 \%$ of previously sedentary and lightly active participants (adults $<30 \mathrm{~min} /$ week, young people $<60 \mathrm{~min} /$ week moderate physical activity) achieved recommended physical activity guidelines. This study reported limitations: a lack of follow-up, the use of moderate physical activity end point averages, difficulties in collecting data at a local level and low numbers of completers $(n=1022)$ compared with those engaged $(\mathrm{n}=10433) .{ }^{14} \mathrm{~A}$ recent House of Lords report attributes weak evidence to a lack of joined-up government thinking about both the relationship between sport, physical activity and health, and the responsibility for ensuring public health outcomes from elite and non-elite sport. ${ }^{15}$ The weak evidence base is also linked to different paradigms influencing sport and health research ${ }^{8}$ and methodological challenges of designing and evaluating complex community interventions for health behaviour change as well as to the limits of theories used. ${ }^{16}$ The Health and Sport Engagement (HASE) Project includes a rigorous comparative evaluation, strong quasi-experimental design and the inclusion of economics to address the identified need for better evaluation of community projects aimed at increasing physical activity through sporting interventions.

The most frequently used theories and models for health behaviour change in policy and practice arise from the field of psychology. However, these approaches have not successfully accounted for the complexities of ecological factors that impact on health behaviours. ${ }^{17}$ Successful community-based health interventions are associated with extensive formative research and a theoretical and practical focus on changing social norms. ${ }^{12}$ This makes empowerment theory a useful theoretical approach for understanding the complexities of raising physical activity levels through community sport. Empowerment theory is focused on social relations of power. Its basic tenet is that the ability of people to take control over their actions is unequal, multidimensional and dynamic and affected by demographic and contextual factors. Proponents of sociological theories of power propose that an understanding of social structures and processes can improve the possibility that people can actively decide on and take control of their lives and thus become empowered. ${ }^{18}$ At the community level, empowerment theory examines people's capacity to influence organisations and institutions which impact on their lives. ${ }^{19} 20$ The theory addresses the processes by which personal and social aspects of life enable and constrain behaviours, and this provides the theoretical basis of this study.

This paper explains the methods used to evaluate the outcomes, costs and processes of designing and delivering a HASE Programme in local community contexts. The study will be the first mixed-method evaluation of a complex community sport intervention aiming to engage inactive people in sustained sporting activity to promote physical activity, health and well-being.

\section{Aim}

The HASE Project is a 3-year community sport development project delivered in the London Borough of Hounslow (LBH) that aims to engage previously inactive people in sustained sporting activity for $1 \times 30 \mathrm{~min}$ a week. The project is a partnership between Brunel University London and the LBH Community Sport and Physical Activity Network (CSPAN). The HASE evaluation question is: "What are the processes, costs and outcomes of designing and delivering a Health and Sport Engagement Programme in local community contexts?"

The intervention functions to educate and support people to take up community sport activities as a way to increase physical activity for the purpose of improving health and well-being, and to develop quality service provision of community sport for inactive people.

\section{THE HASE INTERVENTION}

There are two core overlapping intervention phases, each with activities contributing to the design, delivery and evaluation of the HASE Project. Phase 1 (P1) is a 
6-month planning, training and design phase. Phase 2 (P2)is a 12-month recruitment and delivery phase with staggered starts by a portfolio of community sport for health projects tailored to the needs of inactive target groups. Currently, HASE community sport projects are engaged in $\mathrm{P} 2$ data collection procedures.

In P1, HASE Projects are planned with $\geq 10$ delivery partners for potential pathways of impact between community sport interventions and physical activity, health and well-being outcomes. The intervention is informed by evidence from relevant local and national data sets on inactivity (Sport England Active People Survey and Hounslow Joint Strategic Needs Assessment) and through focus group work with local people to understand the needs of inactive people. Focus group participants are purposively selected from the local population known to be inactive. The tenets of empowerment theory are used in this study to identify inactive participants for whom there are barriers to physical activity and who are likely to benefit from the intervention (eg, older age groups, those living in areas of high deprivation, people with disabilities, minority ethnic populations and young girls), and to tailor the interventions according to the needs of participants. ${ }^{21}$ Empowerment theory provides the basis for discussions and activities about the reasons why people do and do not participate in sport including the sociocultural, environmental, political and economic factors that influence experiences, motives, emotions and perceptions. Focus groups lasting $1 \mathrm{~h}$ will be completed with approximately 6-12 people recruited through partner networks from community groups drawn from demographic profiles significant in targeting and recruiting inactive people. Numbers of focus groups will vary according to the significance of the demographic profile and context of the population to the wider aims of the HASE Project, and accessibility of the population groups through the project networks. Focus groups will use the principles of community participatory research by drawing on the knowledge and expertise of potential participants, delivery personnel and researchers about sport engagement, delivery and evaluation in a collaborative approach based on reciprocal learning and taking action on findings. ${ }^{22}$ The approach aims to maximise the quality of relevant knowledge applicable to developing effective community interventions.

In designing the intervention in P1, HASE deliverers will be contracted to attend a bespoke package of HASE training. Community sport is traditionally delivered by sports coaches whose training does not include detailed knowledge of public health issues or practical approaches to targeting and delivering to inactive people. ${ }^{23}$ HASE training will develop an understanding of public health, and of targeting, recruiting and retaining inactive people to sport for health. Example training includes: The Royal Society for Public Health (RSPH) Level 2 Award in Understanding Health Improvement and workshops on targeting, promoting and retaining inactive people to sport (http://makesportfun.com/; engaging women and girls in sport http://www. streetgames.org); disability, inclusion and sport (http:// www.interactive.uk.net/).

To address issues of continual renewal and engagement of inactive participants, public health professionals whose work involves delivering interventions where sport and physical activity could be included (eg, interventions for weight loss, neurological disorders, mental health support) will attend a bespoke workshop on referring inactive people to community sport (http:// makesportfun.com/). To address the need for crosssector collaboration between local sport and public health groups, sports coaches and public health professionals will attend a bespoke knowledge exchange workshop on getting to know and understand the roles and working practices of personnel in each sector (http:// makesportfun.com/).

In P2, delivery partners will be required to identify inactive target participants and recruit and retain a minimum of 20 previously inactive participants on the basis of what has been learnt in HASE training, capacity building and local evidence of demand. Provision will be tailored to participants' needs. Partners will offer a portfolio of activities at multiple sites. Sport is defined in relation to the European Charter for sport. ${ }^{24}$ Any sport recognised by Sport England can be provided from traditional games (rugby, football, netball), to fitness, dance and meditative activities, and adapted sports for those with physical or mental disabilities. Funding for P1 and P2 will require the submission of a project budget for equipment, facilities, trained coaching staff and volunteers, publicity and participant transport. Partners receive $50 \%$ funding on attending HASE training, submitting a project description and setting up their project for P2 delivery. The remaining $50 \%$ funding is awarded on recruitment of 20 previously inactive participants, completion of outcome measures by the target group and delivery of community sport activities for the first 2 months of P2. Partners will deliver weekly sports sessions over a 12-month period. Where projects cannot engage at least 10 regular participants after the first 2 months of delivery, they will be required to change their delivery model to achieve $\geq 18$ regular participants without which projects will not receive full funding.

\section{Community sport delivery group inclusion criteria}

Established local community sport delivery groups are recruited during $\mathrm{P} 1$ to deliver interventions in $\mathrm{P} 2$. Delivery groups $(n \geq 10)$ are included if they have: (1) a strategic interest in delivering community sport to inactive people and (2) a track record of $\geq 6$ months of delivering community sport.

\section{Targeting participants}

HASE community sport for health interventions will be informed by an understanding of the needs and desires of inactive people who are targeted during P1 and recruited during P2 through methods developed in 
HASE training or already used by deliverers. These will include HASE focus groups, face-to-face presentations, posters, emails, advertisements in local magazines, telephone calls, text messages and taster sessions. HASE sports will be promoted as suitable for previously inactive people. Active participants will be allowed to participate in HASE sports but will not be included in the outcome measurement.

\section{THE HASE EVALUATION}

The HASE evaluation will consist of three parts: assessment of outcomes, cost/cost-effectiveness analysis and process evaluation. Our reporting will use the standardised nomenclature for describing the irreducible components of interventions. ${ }^{25}$

\section{Assessment of outcomes}

Study design and recruitment

The evaluation of outcomes will employ an interrupted time series design to allow comparison of the community sports intervention with no intervention. The data on outcome measures will be collected at multiple points before and after the community sports intervention delivery. ${ }^{26} 27$ Three time points before $(-42,-21,0$ days -immediately before the intervention) and after the intervention $(+21,+42,+63$ days $)$ will be selected, following the Cochrane recommendations. ${ }^{28}$ These time points reflect the recent literature showing that individuals' physical activity intentions are dynamic and fluctuate daily and weekly ${ }^{29}$ and, therefore, that longer periods of assessment are unlikely to show strong associations between HASE interventions and outcomes. However, since questions are often raised about the longterm maintenance of changes in physical activity ${ }^{29} 30 \mathrm{a}$ 12-month follow-up survey will be undertaken.

A formal power analysis to determine the sample size was not conducted as the threshold for raising physical activity levels through community sport interventions is not known. HASE sport projects are designed to achieve target numbers of 20 participants each and ensure an overall target number of 300 previously inactive participants in the HASE Programme. The target number was based on the expectations of achieving "wider engagement' with 3225-3950 inactive people in the LBH and evidence from unpublished local reporting by LBH of a $10 \%$ retention rate for $12-16$ weeks participation in local exercise interventions. 'Wider engagement' was defined by $\mathrm{LBH}$ as unique contacts with individuals through a community service. This includes promotion of the HASE Programme through contacts made in person and by email, text, mail and telephone and was operated through local community, sport delivery and public health stakeholder networks.

\section{Participant inclusion criteria}

Participants must be physically inactive before taking part in HASE sports. As required by Sport England, potential participants will be screened for activity level using the single item physical activity measure. ${ }^{31}$ Activity levels are self-defined by the number of days on which participants report doing $\geq 30 \mathrm{~min}$ physical activity enough to raise the breathing rate (moderate-vigorous physical activity, MVPA). Participants are deemed to be inactive if they report 0 or 1 day of MVPA and written consent into the outcome evaluation will be sought from them. To achieve data points prior to the start of the community sport intervention, they will be linked to two $45 \mathrm{~min}$ 'taster sessions' to include short (20 min) lowlevel activity sessions, talks about sport and health, 'meet the coach' sessions, project descriptions and facility tours. The remaining time points will coincide with delivery sessions (figure 1).

\section{Outcome measures}

Consenting participants will complete a paper questionnaire (HASE Lifestyle Survey; available on request from the corresponding author) at each data collection point, with researchers available to assist participants in reading and understanding the questions and to collect completed questionnaires. Access to translation services will be available through LBH and through community leaders in appropriate forms (printed, verbal and signing for the deaf). Participants who are absent at data collection sessions will be sent a questionnaire by post with a prepaid return envelope.

Questions on outcome measures are based on validated standard questionnaires used in physical activity-related studies to detect changes over time for physical activity levels, ${ }^{32}$ subjective well-being, ${ }^{33}$ and health outcomes and costs. ${ }^{34}$ To supplement outcome measures, attendance data will be collected. HASE sport delivery groups are contracted to submit monthly registers for 12 months post initial delivery and, for those who continue, to project end.

The questionnaires completed prior to delivery of the community sports intervention comprise four sections and take around $15 \mathrm{~min}$ to complete.

- Section A uses the International Physical Activity Questionnaire self-administered short form ${ }^{35}$ (IPAQSF http://www.ipaq.ki.se/ipaq.htm) to record frequency, intensity and duration of physical activity. This will allow reporting of:

- Change in days (that last at least $30 \mathrm{~min}$ ) of selfreported physical activity of (1) any intensity, (2) vigorous intensity and (3) moderate intensity;

- Change in days (that last at least $30 \mathrm{~min}$ ) of selfreported (1) walking, (2) sport and (3) any physical activity.

Since IPAQ-SF does not include a specific question on sport, Sport England designed additional questions on frequency and duration of sport participation to be included in the questionnaire and to support their wider evaluation of projects in the Get Healthy Get Active research programme. 
Figure 1 Study overview (HASE, Health and Sport Engagement).

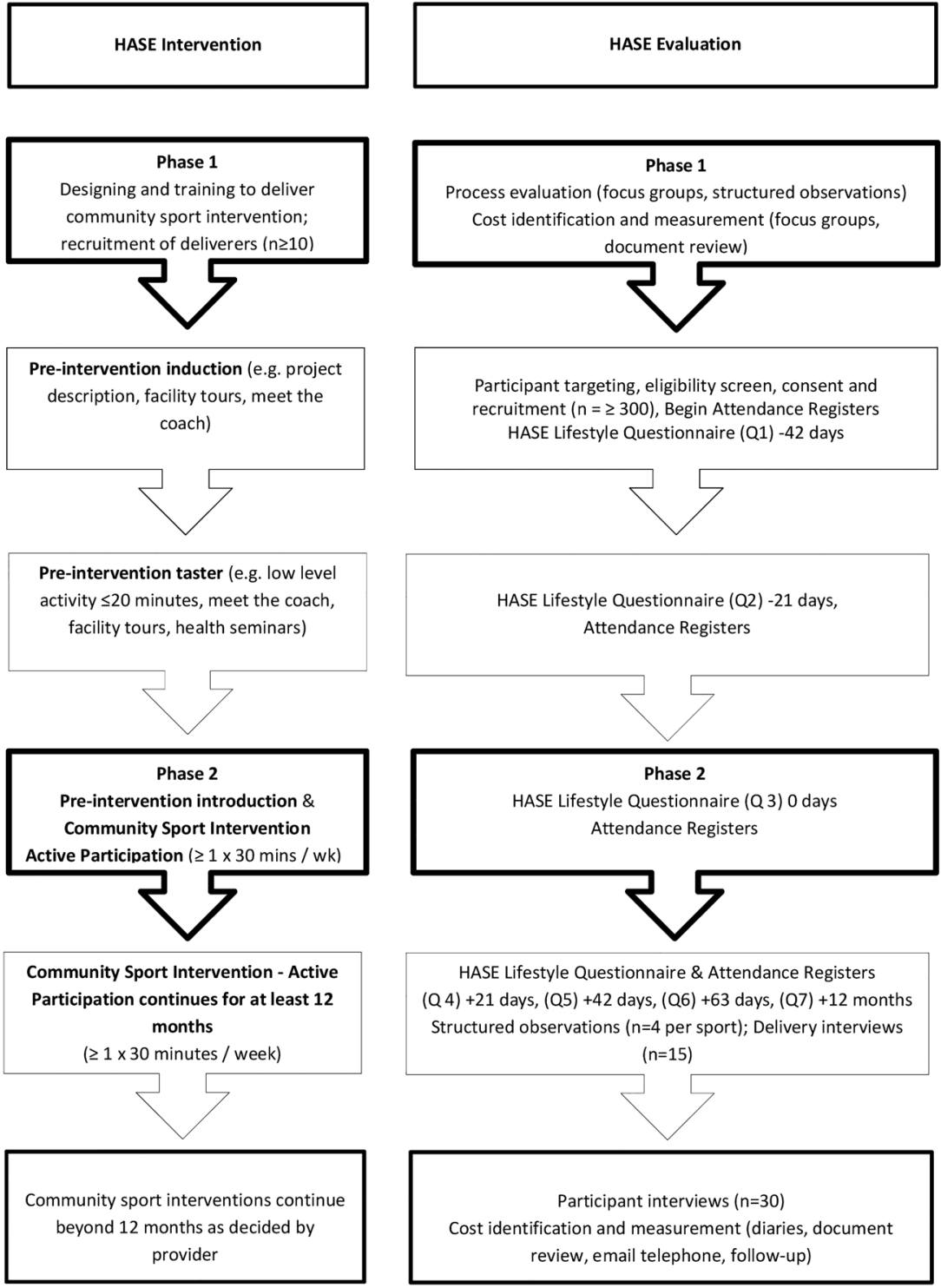

Participant targeting, eligibility screen, consent and uitment $(n=\geq 300$ ), Begin Attendance Registers

ASE Lifestyle Questionnaire (Q 3) 0 days Atendance Registers

The questionnaires completed during delivery of the community sports intervention contain an additional section with questions on travel time and out-of-pocket expenses associated with attendance of the taster and community sport sessions.

\section{Statistical analysis of outcomes}

Data from the questionnaires will be linked through unique individual study identification numbers allocated and printed on each questionnaire. Descriptive statistics, including measures of central tendency, will be provided for outcomes and participant costs before and after the community sports intervention. Patterns of missing data will be examined, with approaches for replacing missing data to include regression-based imputation and indicator methods, determined by type, and size of data (indicating variable in question). ${ }^{39} 40$

Regression analyses controlling for participant characteristics will estimate changes in the level and trend in physical activity, quality of life, well-being and participant costs following introduction of the 
community sports intervention. This will compare the difference in costs predelivery and postdelivery of the community sport intervention, with the difference in number of days of physical activity (any intensity and type) lasting at least $30 \mathrm{~min}$ and EQ-5D-based quality-adjusted life years (QALYs) for the community sports intervention versus no intervention. In addition, temporal trends in outcome measures will be assessed by comparing the differences predelivery and postdelivery of the community sport intervention and 12 months postdelivery of the intervention (12 months post the first session for community sports intervention) for: (1) number of days of physical activity (any intensity and type) totalling at least $30 \mathrm{~min}$; (2) subjective well-being and (3) quality of life. Measurements will be summarised as the mean and 95\% CI. We expect such analysis to include segmented regression models. ${ }^{41}$ Autocorrelation will be tested and accounted for using standard approaches. ${ }^{26}$

\section{Resource use, cost and cost-effectiveness analysis}

The main objective of the economic analysis is to assess the cost-effectiveness of adding on the delivery of community sport interventions compared with no community sport intervention among inactive people. The analysis will be conducted from a societal perspective (including the National Health Service (NHS), Local Authority, 3rd sector, participants, private sector, Sport England and University). The secondary objective is to describe the costs that lie behind the level of physical activity maintenance 12 months postdelivery. Resource use data will be collected for the organisations involved.

Resource use will be identified, by delivery organisation, for: (1) set-up and design of the 15 HASE community sports programmes; (2) training of coaches; (3) recruitment of participants and (4) running of taster and community sport interventions. Identification of who did what, to whom, where and how often for (1) and (2) will be based on interviews and review of project records with the principal investigator (PI). Identification for (3) and (4) will be developed in two stages; first, through a workshop with community sport deliverers on key resources ensuring coverage of highcost items or frequently incurred small costs associated with their activities including in-kind contributions, ${ }^{42}$ and second, by face-to-face interviews with each deliverer, recorded and transcribed for accuracy The description of the programme will be reviewed by the programme deliverers. Measurement of the physical quantities of resource use will include: completion and review of diaries; review of invoices and administrative records; and interviews alongside email and telephone contact with designers and deliverers.

In addition to costs of provision, participant-level resource use data will be collected for out-of-pocket expenses in section B of the HASE Lifestyle Survey. These resource uses have been shown to increase the private costs of physical activity and impact on costeffectiveness of physical activity interventions. ${ }^{43} 44$

Unit costs will be valued using national averages (eg, PSSRU 2013 ${ }^{45}$ to increase their generalisability. All costs will be expressed in pounds sterling, inflated to the base year where appropriate. Since the period of study is less than 1 year, costs will not be discounted. Each sports programme will be costed separately and apportioned to participants as appropriate. EQ-5D-5L QALYs will be weighted using the 'crosswalk' methodology with UK values ${ }^{46}$ or the new value set for England ${ }^{47}{ }^{48}$ if available in time.

Costs will be compared with outcome measures. Total and incremental costs and effects will be presented using a fuller distributional description, given the expected skewness and 'lumpiness' of costs attributed to individuals attending different sport interventions. Incremental costs and effects will also be estimated using regression models. If the community sports intervention has convincing evidence of dominance over no intervention, then further economic analysis will not be undertaken. In the case of no dominance, incremental cost-effectiveness ratios (ICERs) will compare the differences in costs and outcomes between community sport intervention and no intervention. To reflect sampling variation and uncertainties in the cost-effectiveness threshold values, results will also be presented using cost-effectiveness planes and cost-effectiveness acceptability curves. Deterministic sensitivity analysis will explore: (1) fixed variables within the economic analysis (eg, discount rate); (2) other policy control variables (eg, who provides intervention); (3) alternative perspectives; (4) likely cost of the national roll-out using scenario analyses and (5) others as information arises from the process evaluation.

\section{Process evaluation}

The process evaluation will examine the efficacy of the HASE Project for achieving its wider aim of engaging previously inactive people in sustained sporting activity for $1 \times 30 \mathrm{~min}$ a week. Process evaluations can include both qualitative and quantitative methods and have particular value in multisite projects where the same interventions are tailored to specific contexts and delivered and received in different ways. ${ }^{49}$ Such evaluations can complement research designs that assess effectiveness and efficiency quantitatively. ${ }^{50}$

The process evaluation will identify where and why interventions are considered successful or not and how best to optimise success so that the study findings can effectively inform policy and practice in community sport. It will evaluate the design, implementation, mechanisms of impact and contextual factors that create different intervention effects in this study in order to develop an optimal community sport intervention and to contribute to decision-making about whether it is feasible to proceed to a larger scale trial by complementing the outcome and economic evaluation. ${ }^{6}$ 


\section{Methods}

This process evaluation will be longitudinal and employ qualitative focus groups, structured observations and in-depth interview methods. It follows established guidance by focusing on the following process factors: fidelity - consistency of the planned interventions; adaptations -changes required for success in different contexts; dose-how much of the intervention is delivered; reachthe degree to which target participants engage with the intervention; acceptability - the extent to which potential participants are willing to receive and engage in the intervention; resources-required to produce successful interventions; mechanisms of impact-including participant and delivery personnel experience, and intended and unintended consequences; and contextual factorswider social factors which strengthen and/or weaken the intervention effects, for example, organisational structures, skill set of delivery personnel, personal circumstances of participants and intervention location. ${ }^{649} 51$ Critical peer review of data collection will ensure that close observations by those most invested in the project are balanced with the level of independence required for high-quality evidence production. ${ }^{51}$

Qualitative focus groups will be employed as both part of the intervention, that is, to understand the community sport needs of inactive people, and in evaluating the efficacy of HASE in designing and delivering an effective intervention. As well as discussing the reasons why people do and do not participate in sport and physical activity, the theoretical framework of empowerment will allow for examination of participant views about design and delivery of community sport to engage inactive people. The data will be used to evaluate the fidelity, adaptations, dose, reach and acceptability, mechanisms of impact and contextual factors that contribute to the success and failure of community sport interventions.

Structured observations of all HASE training and planning sessions will be conducted. Structured observations of the implementation (delivery) of each HASE community sport project will be conducted on four occasions at 3-month intervals during the initial 12-month delivery phase by the HASE PI and research assistants. Delivery groups receive notification of observations 1 week in advance. Detailed observation notes are recorded using project-specific observation data sheets designed to enable description of the organisation, timing, equipment, facilities, places and spaces, people, behaviours and emotions involved in the activities being delivered and to reflect the process factors identified above. Thirty minute telephone 'delivery interviews' are conducted with each HASE sport delivery lead $(n=15)$. These determine the precise mechanisms of the design and implementation processes, identify project resources and examine the features of community sport that might lead to long-term sustainable delivery for health and well-being outcomes. Further in-depth, face-to-face 'resource interviews' will be conducted with HASE Project leads and the HASE PI in P2 to obtain detailed information about the monetary and non- monetary costs and resource use associated with design and delivery of HASE sports $(n=15)$. In-depth participant interviews $(n=30)$ will take place to examine the experiences of previously inactive people involved in the community sport intervention.

\section{Analysis of qualitative data}

Interviews will be recorded using an Olympus LS 11 recorder and transcribed ad verbatim. Qualitative data from the process evaluation will be analysed via NVivo 10 software using the principles of thematic analysis. Thematic analysis allows the organisation, detailed description and analysis of patterns of meaning in qualitative data ${ }^{52}$ Qualitative data collection and data analysis will take place concurrently to ensure that outcome, process and economic evaluations are effectively complementary and to enable the production of interim reporting to inform policy and practice. ${ }^{53}$ Analysis will involve repeated reading of observation notes, interview and focus group transcripts to determine the details of the data and to enable researchers to identify key themes and patterns in it. ${ }^{54}$ Themes will be identified by theoretical approaches focused on our analytical interest in empowerment in community sport interventions, and by inductive (data-driven) approaches drawing directly from the data produced. Coding frameworks will be devised to reflect the theoretical focus of the project and the research questions as well as salient issues evident in the data to support the process of identifying, refining and interpreting key themes. ${ }^{55}$

\section{DISSEMINATION}

Participants receive detailed written participant information and written consent is sought. They will be informed of their right to withdraw at any time, without penalty and to anonymity and confidentiality. The study findings will be disseminated through peer-reviewed publications, scientific conferences and knowledge exchange events organised by Sport England and national organisations developing policy on physical activity, and through practice-based case studies.

Acknowledgements The HASE Project is being designed, delivered and evaluated in the London Borough of Hounslow with key delivery partners: Brentford Football Club Community Sport Trust, CB Hounslow United, Cattaway Tennis, Cycle Experience, Centre for Workplace and Community Health at St Mary's University Twickenham, Fusion Lifestyle, England Netball, Hounslow Homes, LKFitness, Sport Impact, The Urban Youth Network and Westside Basketball Club. Additional design and delivery support is from British Airways, British Army Welfare, Cranford Community School, Hounslow and Richmond Community Health, Integrated Neurological Services

(Richmond), UK Dodgeball Association, Make Sport Fun, West Thames College, The Hub Youth Club.

Funding This work is funded by Sport England's Get Healthy Get Active awards.

Contributors LM, TK, NA and JF-R contributed to the design of the HASE Project in collaboration with the project partners. All the authors contributed to the development of the study protocol and drafted, critically appraised and approved the final manuscript. 
Competing interests The HASE intervention is co-developed by Brunel University investigators, LBH, local community participants and sport deliverers. Brunel University investigators will evaluate the project.

Ethics approval This study has been approved by Brunel University Research Ethics Committee, Division of Sport, Health and Exercise (reference number RE33-12)

Provenance and peer review Not commissioned; externally peer reviewed.

Open Access This is an Open Access article distributed in accordance with the Creative Commons Attribution Non Commercial (CC BY-NC 4.0) license, which permits others to distribute, remix, adapt, build upon this work noncommercially, and license their derivative works on different terms, provided the original work is properly cited and the use is non-commercial. See: http:// creativecommons.org/licenses/by-nc/4.0/

\section{REFERENCES}

1. Sport England. Creating a sporting habit for life: a new youth sport strategy. London: DCMS, 2012.

2. Department of Culture Media and Sport. Before, during and after: making the most of the London 2012 games. London: Crown Copyright, 2008.

3. Sport England. The active people survey. http://archive sportengland.org/research/active_people_survey.aspx (accessed 8 Jan 2015)

4. Medical Research Council. Developing and evaluating complex interventions: new guidance. London: Medical Research Council, 2008.

5. Campbell M, Fitzpatrick R, Haines A, et al. Framework for design and evaluation of complex interventions to improve health. BMJ 2000;321:694-6.

6. Campbell NC, Murray E, Darbyshire J, et al. Designing and evaluating complex interventions to improve health care. BMJ 2007;334:455

7. Priest N, Armstrong R, Doyle J, et al. Interventions implemented through sporting organisations for increasing participation in sport. Cochrane Database Syst Rev 2008;16:CD004812.

8. Cavill N, Richardson D, Foster C. Improving health through participation in sport: a review of research and practice. Oxford: BHF Health Promotion Research Group, University of Oxford, 2012.

9. Lupton B, Fønnebø V, Søgaard A. The Finnmark Intervention Study: is it possible to change CVD risk factors by community-based intervention in an Arctic village in crisis? Scand $J$ Public Health 2003;31:178-86.

10. Brown W, Eakin E, Mummery K, et al. 10,000 steps Rockhampton: establishing a multi-strategy physical activity promotion project in a community. Health Promot J Austr 2003;14:95-100.

11. Wendel-Vos G, Dutman A, Verschuren W, et al. Lifestyle factors of a five-year community-intervention program: the Hartslag Limburg intervention. Am J Prev Med 2009;37:50-6.

12. Merzel C, D'afflitti J. Reconsidering community-based health promotion: promise, performance, and potential. Am J Public Health 2003:93:557-74.

13. Roussos ST, Fawcett SB. A review of collaborative partnerships as a strategy for improving community health. Annu Rev Public Health 2000;21:369-402.

14. Pringle A, Gilson N, McKenna J, et al. An evaluation of the local exercise action pilots and impact on moderate physical activity. Health Educ J 2009;68:179-85.

15. House of Lords Select Committee on Science and Technology 1st Report of Sessions 2012-13. Sport and exercise sciences and medicine: building on the Olympic legacy to improve the nation's health. London: The Stationary Office, 2013.

16. Ogilvie D, Bull F, Powell J, et al. An applied ecological framework for evaluating infrastructure to promote walking and cycling: the iConnect study. Am J Public Health 2011;101:473-81.

17. Linke SE, Robinson CJ, Pekmezi D. Applying psychological theories to promote healthy lifestyles. Am J Lifestyle Med 2013;1559827613487496.

18. Tomlinson A. POWER: domination, negotiation, and resistance in sports cultures. J Sport Soc Issues 1998;22:235-40.

19. Lord J, Hutchison P. The process of empowerment: implications for theory and practice. Can J Commun Ment Health 1993;12:5-22.

20. Fawcett SB, Paine-Andrews A, Francisco VT, et al. Using empowerment theory in collaborative partnerships for community health and development. Am J Community Psychol 1995;23:677-97.

21. Michie S, Prestwich A. Are interventions theory-based? Development of a theory coding scheme. Health Psychol 2010;29:1-8.
22. Macaulay AC, Commanda LE, Freeman WL, et al. Participatory research maximises community and lay involvement. BMJ 1999;319:774

23. Almand L, Almand M, Saunders, L. Coaching sport for health: a review of literature. London: Sports Coach UK, 2013.

24. UNESCO European Charter for Sport 1992. http://portal.unesco.org/ education/en/ev.php-URL_ID=2221\&URL DO=DO TOPIC\&URL SECTION=201.htm

25. Michie S, Richardson M, Johnston M, et al. The behavior change technique taxonomy (v1) of 93 hierarchically clustered techniques: building an international consensus for the reporting of behavior change interventions. Ann Behav Med 2013;46: 81-95.

26. Ramsay CR, Matowe L, Grilli R, et al. Interrupted time series designs in health technology assessment: lessons from two systematic reviews of behaviour change strategies. Int J Technol Assess Health Care 2003;19:613-23.

27. Pape UJ, Millett C, Lee JT, et al. Disentangling secular trends and policy impacts in health studies: use of interrupted time series analysis. J $R$ Soc Med 2013;106:124-9.

28. Cochrane Effective Practice and Organisation of Care Group. Including Interrupted Time Series (ITS) designs in a EPOC review. 1998. http://epoc.cochrane.org/sites/epoc.cochrane.org/files/uploads/ inttime.pdf (accessed 20 Jun 2014).

29. Conroy DE, Elavsky S, Hyde AL, et al. The dynamic nature of physical activity intentions: a within-person perspective on intention-behavior coupling. J Sport Exerc Psychol 2011;33:807.

30. Fjeldsoe B, Neuhaus M, Winkler E, et al. Systematic review of maintenance of behavior change following physical activity and dietary interventions. Health Psychol 2011;30:99-109.

31. Milton K, Bull F, Bauman A. Reliability and validity testing of a single-item physical activity measure. Br J Sports Med 2011;45:203-8.

32. Fuller NR, Williams $\mathrm{K}$, Shrestha $\mathrm{R}$, et al. Changes in physical activity during a weight loss intervention and follow-up: a randomized controlled trial. Clin Obes 2014;4:127-35.

33. Evans J, Macrory I, Randall C. Measuring national well-being: life in the UK, office for 34. London: National Statistics, 2015.

34. Harris T, Kerry SM, Victor CR, et al. PACE-UP (Pedometer and consultation evaluation-UP) - a pedometer-based walking intervention with and without practice nurse support in primary care patients aged 45-75 years: study protocol for a randomised controlled trial. Trials 2013;14:418-20.

35. Craig CL, Marshall AL, Sjöström M, et al. international Physical Activity Questionnaire: 12-country reliability and validity. Med Sci Sports Exerc 2003;195:3508-1381.

36. Anokye N, Pokhrel S, Fox-Rushby J. Economic analysis of participation in physical activity in England: implications for health policy. Int J Behav Nutr Phys Act 2014;11:117-20.

37. Herdman M, Gudex C, Lloyd A, et al. Development and preliminary testing of the new five-level version of EQ-5D (EQ-5D-5L). Qual Life Res 2011;20:1727-36.

38. Office of National Statistics. Analysis of experimental subjective wellbeing data from the annual population survey. 2011. http://www. ons.gov.uk/ons/rel/wellbeing/measuring-subjective-wellbeing-in-the-uk/ analysis-of-experimental-subjective-well-being-data-from-the-annualpopulation-survey-april-september-2011/report-april-to-september2011.html (accessed 20 Jun 2014).

39. Petrou S, Kupek E. Social capital and its relationship with measures of health status: evidence from the Health Survey for England 2003. Health Econ 2008;17:127-43.

40. Morris S, Sutton M, Gravelle H. Inequity and inequality in the use of health care in England: an empirical investigation. Soc Sci Med 2005:60:1251-66.

41. Wagner AK, Soumerai SB, Zhang F, et al. Segmented regression analysis of interrupted time series studies in medication use research. J Clin Pharm Ther 2002:27:299-309.

42. O'Mara-Eves A, Brunton G, McDaid D, et al. Community engagement to reduce inequalities in health: a systematic review, meta-analysis and economic analysis. Public Health Res 2013;1.

43. Isaacs AJ, Critchley JA, Tai SS, et al. Exercise evaluation randomised trial (EXERT): a randomised trial comparing GP referral for leisure centre-based exercise, community-based walking and advice only. Health Technol Assess 2007;11:1-165.

44. Anokye NK, Trueman P, Green C, et al. The cost-effectiveness of exercise referral schemes. BMC Public Health 2011;11:954.

45. Curtis LA. Unit costs for health and social care Personal Social Services Research Unit (PSSRU). Canterbury: University of Kent, 2013.

46. Van Hout B, Janssen MF, Fen YS, et al. Interim Scoring for the EQ-5D-5L: mapping the EQ-5D-5L to EQ-5D-3L value sets. Value Health 2012;15:708-15. 
47. Oppe M, Devlin N, van Hout B, et al. A program of methodological research to arrive at the new international EQ-5D-5L valuation protocol. Value Health 2014;17:445-53.

48. Devlin N, Van Hout B. An EQ-5D-5L value set for England OHE Seminar Oct 3rd 2014. http://www.slideshare.net/OHENews/ ohe-seminar-5-I-value-set-oct2014

49. Oakley A, Strange V, Bonell C, et al. Process evaluation in randomised controlled trials of complex interventions. $B M J$ 2006;332:413.

50. Wall M, Hayes R, Moore D, et al. Evaluation of community level interventions to address social and structural determinants of health: a cluster randomised controlled trial. BMC Public Health 2009;9:207.
51. Moore G, Audrey S, Barker M, et al. Process evaluation in complex public health intervention studies: the need for guidance. J Epidemiol Community Health 2014;68:101-2.

52. Braun V, Clarke V. Using thematic analysis in psychology. Qual Res Psychol 2006;3:77-101.

53. Miles MB, Huberman MA. Qualitative data analysis: an expanded sourcebook. London: Sage, 1994.

54. Sparkes AC, Smith B. Qualitative research methods in sport, exercise and health: from process to product. London: Routledge, 2013.

55. Attride-Stirling J. Thematic networks: an analytic tool for qualitative research. Qual Res 2001;1:385-405. 